\section{Cost of brain disorders}

SIR - We were surprised to read ( $\mathrm{Na}$ ture 357,$348 ; 1992$ ) the statement that "there is as yet no certain link between significant progress in understanding neuronal function and its application to any human disease".

In the half a century since chemical neurotransmission was established as the major mode of communication between neurons, we have seen the introduction of many therapies. A few examples of human diseases where totally new treatments have resulted directly from this fundamental discovery include hypertension, myasthenia gravis, Parkinson's disease, migraine and epilepsy.

Diseases where treatments have been refined as a result of discoveries about neuronal function include depression and schizophrenia, and novel treatments for these and other diseases of the nervous system are likely to follow from current research. Neuroimmunology and molecular neurogenetics are two other areas that have had a significant influence on neurological disease.

Not content with writing something that could be misused by those who are against science, you go on to argue purely in terms of economics that providing more money for research into diseases such as Alzheimer's would lead, if a successful treatment were discovered, to a loss of jobs by those constructing nursing homes and by hospital workers and care-givers.

Are you suggesting that society would choose to condemn sufferers and their relatives to years of misery rather than solve a problem in the management of health care? It would have been much more appropriate for a journal that is sponsoring an international conference on 'The brain in well-being and disease' to support neuroscientists in their endeavours to unravel the workings of the brain so that those who have diseases that affect the very essence of our human nature can have some hope that their terrible suffering will be relieved.

F. E. Bloom (Scripps Research Institute, La Jolla, California, USA); A. Cowey (University Department of Experimental Psychology, Oxford, UK); R. L. M. Faull (Department of Anatomy, School of Medicine, University of Auckland, New Zealand); D. G. Grahame-Smith (University Department of Clinical Pharmacology, Radcliffe Infirmary, Oxford, UK); J. A. Gray (Department of Psychology, Institute of Psychiatry, Maudsley Hospital, London, UK); T. Hökfelt (Department of Histology, Karolinska Institute, Stockholm, Sweden); L. L. Iversen (Neuroscience Research Centre, Merck Sharp \& Dohme Research Laboratories, Harlow, UK); R. R. LIInas
(Department of Physiology and Biophysics, NYU Medical Center, New York, USA); C. D. Marsden (Institute of Neurology, National Hospital, Queen's Square, London, UK); D. P. Purpura (Albert Einstein College of Medicine, Bronx, New York, USA); M. C. Raff (Department of Biology, University College, London, UK); A. D. Smith (University Department of Pharmacology, Oxford, UK); S. H. Snyder (Department of Neuroscience, Johns Hopkins University School of Medicine, Baltimore, Maryland, USA); P. Somogyi (MRC Anatomical Neuropharmacology Unit, Oxford University, Oxford, UK)

SIR - I was distressed to read the leading article disparaging the report The Costs of Disorders of the Brain. This report argued that the estimated $\$ 401$ billion annual costs for brain disorders in the United States justifies a substantial increase in funding for brain research. Nature did not contest the validity of these cost estimates, which represent 7.3 per cent of the US gross national product.

Your first argument states that "there is as yet no certain link between significant progress in understanding neuronal function and its application to any human disease". This statement is antiscientific and at variance with the substantive advances in our appreciation of brain mechanisms in such common disorders as senile dementia of the Alzheimer's type, schizophrenia and substance abuse. Two decades ago, these were not even recognized as brain diseases, whereas there is now substantial understanding of their molecular and cellular pathology, which has implications for treatment.

Your second argument that curing these disorders will result in loss of employment in the health industry involved in their treatment suggests that we should resume the Cold War to maintain the defence industries. But medical care, like military expenditure, is not a productive use of capital.

The major point of the report was that the investment in brain research is not proportionate to the investment in research on cancer and cardiovascular diseases when the costs to society of the diseases are considered.

This inequity reflects the historical stigma associated with psychiatric, neurological and substance abuse disorders coupled with our past ignorance of brain mechanisms involved in these disorders. The recent rapid advances in neuroscience certainly justify a reappraisal of funding commitments that recognizes the current state of know- ledge and the costs to society of brain disorders.

Joseph T. Coyle

(President)

Society for Neuroscience,

11 Dupont Circle, NW, Suite 500 ,

Washington, DC 20036, USA

Our case was simply that, among the "more than enough reasons to ask federal agencies to contribute large sums to brain research", that given ("that the science right now is ripe for it") is more cogent than that based on the gross cost of disorders of the nervous system. - Editor, Nature.

\section{No access}

SIR - Whether by intent or serendipity, there was a remarkable and sobering piece of editorial selection in Nature for 30 April 1992.

The leading article, "Does science leave room for soul?" (356, 729; 1992) was dispiriting for a variety of reasons, especially because we have all hoped that the anti-intellectualism that it discussed (as well as an imminent fear of the complexity of science) had largely been exorcised by modern education. Clearly and frighteningly, this is far from the case.

Donald Hayes's “The growing inaccessibility of science" $(\mathbf{3 5 6}, \mathbf{7 3 9} ; \mathbf{1 9 9 2 )}$ provided part of the explanation. When fewer and fewer scientists can understand one another - and when most of us can come to grips with only a tiny fraction of the Letters to Nature - how can the public at large be expected to understand science? And if they cannot understand it, how can it possibly contribute to the elucidation of their world? Is it then any wonder that so many people are turning to religious fundamentalism, astrology and other such duplicitous practices? That development itself would be bad enough, but a few other alarming thoughts follow.

If the public devalues science, how long - as the leading article adumbrated - can we expect that electors will continue to tolerate government funding of our activities? And, indeed, if the mass of detailed science is meagrely accessible to scientists generally, how is new scientific work to be assessed and deceit prevented? Such attacks on the future of science portend a dark age. Are scientists willing to step boldly into the "public culture" and explain our work cogently and lucidly? If we are not, do we deserve a future?

\section{John Carmody}

School of Physiology \& Pharmacology, University of New South Wales,

PO Box 1, Kensington,

NSW 2033, Australia 